\title{
Adverbialpositionen im deutsch-englischen Vergleich
}

\author{
Werner Frey \& Karin Pittner, Stuttgart \\ In: \\ M. Doherty (ed.), Sprachspezifische Aspekte der Informationsverteilung. Berlin: Akademie-Verlag, \\ 14-41. (Reihe studia grammatica)
}

Abstract

Der Ausgangspunkt ist die These, daß die verschiedenen Adverbialklassen im Deutschen unterschiedliche Basispositionen aufweisen und daß sich diese durch unterschiedliche strukturelle Anforderungen an die Klassen ergeben. Es soll gezeigt werden, daß sich die plausible Vermutung, daß die Adverbialklassen in Sprachen wie dem Deutschen und dem Englischen entsprechenden strukturellen Bedingungen unterliegen, bestätigt.

Unterschiede im Verhalten der Adverbiale in den beiden Sprachen werden demnach nicht durch unterschiedliche Eigenschaften der Adverbiale erfaßt, sondern diese ergeben sich durch die unterschiedlichen Satzstrukturen und die unterschiedlichen Weisen der Argumentverwaltung. Dies wird illustriert anhand von Adverbialen der Art und Weise, Lokal- und Temporaladverbialen, Adverbialen der Subjekthaltung und Satzadverbialen.

\section{Einleitung}

In Frey/Pittner (i.E.) haben wir einen Versuch unternommen, die Regularitäten der Stellung von Adverbialen im Mittelfeld des deutschen Satzes zu formulieren. Dabei war unser Ziel, die folgenden Annahmen zu untermauern:

es läßt sich nachweisen, daß nicht nur den Komplementen des Verbs, sondern auch adverbialen Adjunkten eine Grundposition im Mittelfeld zukommt

die Grundpositionen von Adverbialen spiegeln ihre semantischen Beziehungen zum Restsatz wider

wie die Verbargumente müssen Adverbiale nicht an ihrer Grundposition auftreten, sondern können gescrambelt werden

Um die Grundpositionen zu ermitteln, haben wir eine Reihe von Tests verwendet. ${ }^{1}$ Aufgrund der Anwendung dieser Tests sind wir zu dem Ergebnis gelangt, daß die adverbialen Adjunkte in 5 syntaktisch relevante Klassen fallen, nämlich Frame- und Bereichsadverbiale, Satzadverbiale, ereignisbezogene Adverbiale, ereignisinterne Adverbiale und prozeßbezogene Adverbiale. ${ }^{2}$

(1) gibt einige Beispiele für die Zuordnung der verschiedenen Adverbialtypen zu diesen Klassen:

Adverbialklassen

prozeßbezogene Adverbiale: ereignisinterne Adverbiale:

ereignisbezogene Adverbiale: propositionsbezogene Adverbiale: Frameadverbiale:

\author{
Adverbiale der Art und Weise \\ Instrument und Komitativ, Lokaladverbiale, Adverbiale der \\ Subjekthaltung \\ Temporaladverbiale, Kausaladverbiale \\ Satzadverbiale \\ den Geltungsbereich von Propositionen einschränkender \\ Gebrauch von Lokal- und Temporaladverbialen
}

1 Diese Tests sind (a) Fokusprojektionstest, (b) Thema-Rhema-Bedingung von Lenerz (1977), (c) Skopustest (nach Frey 1993), (d) Komplexes Vorfeld, (e) Stellungsfestigkeit indefiniter w-Pronomina und (f) Prinzip-C-Effekte. S. Frey/Pittner (i.E.) für eine Beschreibung und Anwendung dieser Tests. Sprechaktbezogene Adverbiale wie offen gestanden, um es ganz deutlich zu sagen haben wir wegen ihres Parenthesecharakters nicht berücksichtigt. 
Wir haben argumentiert, daß für die Grundpositionen der verschiedenen Adverbialklassen im deutschen Mittelfeld die folgenden Bedingungen gelten:

(2) Die Abfolge der Adverbiale:

(i) Frame- und Bereichsadverbiale: k-kommandieren die Grundposition der Satzadverbiale

(ii) Satzadverbiale: k-kommandieren das finite Verb und die Grundposition der ereignisbezogenen Adverbiale

(iii) ereignisbezogene Adverbiale: k-kommandieren die Grundposition des ranghöchsten Arguments und die Grundpositionen ereignisinterner Adverbiale

(iv) ereignisinterne Adverbiale: sie werden von ihrem "Bezugsargument" minimal (bezüglich der anderen Argumente) k-kommandiert

(v) prozeßbezogene Adverbiale: k-kommandieren minimal das Verb bzw. den Prädikatskomplex

Ein weiterer Punkt unserer Überlegungen war, daß zwischen den Adverbialen, die zur gleichen Klasse gehören, keine syntaktisch determinierte Grundabfolge vorliegt, sondern daß eventuelle Abfolgepräferenzen rein semantisch determiniert sind. Zwischen Adverbialen, die zu verschiedenen Klassen gehören, liegt dagegen eine durch die Syntax festgelegte Abfolge vor, die allerdings durch Scrambling im Deutschen an der Oberfläche verändert werden kann. Während somit Scrambling von Adverbialen verschiedener Klassen untereinander oder bezüglich der Verbargumente im Deutschen möglich ist, gibt es für die Abfolge der Adverbiale der gleichen Klasse lediglich semantische Präferenzen, sie ist nicht durch die Syntax determiniert. Daher muß die Interpretation in diesem Fall immer der Anordnung an der Oberfläche folgen.

In diesem Aufsatz wollen wir einige Überlegungen anstellen, inwieweit sich die Bedingungen in (2) für die Adverbialpositionen im Englischen bestätigen. Dies soll ein erster Schritt sein, um zu zeigen, daß diese Bedingungen nicht sprachspezifisch sind, sondern, da sie semantische Hierarchieverhältnisse widerspiegeln, übereinzelsprachlichen Charakter haben. Dabei ist natürlich zu erwarten, daß sich die Bedingungen im Englischen aufgrund der anderen Satzstruktur teilweise auf andere Weise manifestieren als im Deutschen.

Eine umfassende Darstellung der Regularitäten der Stellung von Adverbialen im Englischen würde den Rahmen dieses Aufsatzes sprengen. Wir werden uns daher im folgenden auf einzelne Adverbialtypen beschränken.

\section{Die VP-Schalen-Struktur des englischen Satzes}

Seit Larson (1988) nehmen die meisten Syntaktiker an, daß der englische Satz eine binäre, rechtsverzeigende Struktur aufweist. Die folgenden Daten zeigen, daß ein vorangehendes Argument ein nachfolgendes asymmetrisch k-kommandiert, denn die Bindung eines Pronomens durch eine quantifizierte Phrase oder die Lizenzierung eines negativen Polaritätselements wie any erfolgt unter K-Kommando.

(3) a. I gave every worker his paycheck

b. *I gave its owner every paycheck

c. I gave every check to its owner

d. ??I gave his paycheck to every worker

e. I showed no one anything

f. *I showed anyone nothing

g. I sent no present to any of the children

h. *I sent any of the packages to none of the children 
Nun ist Englisch eine VO-Sprache. Nach allem, was man über die syntaktische Strukturierung weiß, ist das folgende keine mögliche Strukturzuweisung für eine VO-Sprache:

\section{(4) $\quad[\mathrm{V}[\mathrm{XP}[\mathrm{YP}[\mathrm{ZP}]]]$}

Diese Struktur ist zwar rechtsverzeigend, aber sie ist nicht endozentrisch. Sie wird von keinem Kopf aufgespannt. V befindet sich nicht am Fuß der Struktur, sondern an der Spitze.

Rechtsverzweigung und VO-Eigenschaft einer Sprache ergeben vielmehr die Notwendigkeit der sog. VPSchalen-Analyse (Larson 1988). Dies bedeutet, daß das Verb eine Kopf-Kette bildet, wobei die verschiedenen Glieder der Kette jeweils eine VP-Schale aufspannen. Damit ergibt sich die binäre Rechtsverzweigung und die Endozentrizität der Strukturen bei gleichzeitiger Präzedenz des Verbs auf der Oberfläche. Wir nehmen an, daß sich alle Argumente in der gleichen strukturellen Position befinden. Alle Argumente haben ihre Grundposition in einer Spec-Position:

$$
\left[\mathrm{V}_{1}\left[\mathrm{VP} \mathrm{XP}\left[\mathrm{t}_{1}{ }^{\prime \prime}\left[\mathrm{VP} \text { YP }\left[\mathrm{t}_{1}{ }^{\prime}\left[\mathrm{VP} \mathrm{ZP}\left[\mathrm{t}_{1}\right]\right]\right]\right]\right]\right]\right]
$$

Der Trigger für diese Satzstruktur ist der Umstand, daß das Verb im Englischen seine Objekte nach rechts lizenziert. Das englische Verb regiert nach rechts und muß daher aus seiner Grundposition wegbewegt werden, um eine Position zu erreichen, von wo aus es die Lizenz erteilen kann.

Nun stellt sich die Frage, wie die Verhältnisse in einer OV-Sprache wie dem Deutschen sind. Man kann analog zu den Daten in (3) zeigen, daß auch das deutsche Mittelfeld binär rechtsverzweigend strukturiert ist. In einer OV-Sprache regiert aber das Verb nach links und muß daher seine Grundposition nicht verlassen, um seinen Argumenten die Lizenz zu erteilen:

(6) $\quad[\mathrm{XP}[\mathrm{YP}[\mathrm{ZP} \mathrm{V}]]]$

(6) ist rechtsverzweigend und erfüllt die Forderung der Endozentrizität, ohne daß eine Kopfkette notwendig wäre.

Bei Larson (1988) ist die Grundposition des Subjekts in der Spec-Position der obersten VP-Schale. Einige Autoren (z.B. Bowers 1993, Chomsky 1995, Ernst 1998a) vertreten einen modifizierten Standpunkt. Sie nehmen an, daß sich die Grundposition des Subjekts im Englischen in der Spec-Position einer anderen Projektion befindet, die direkt über der VP-Schalenstruktur verortet wird. Bowers nennt diese Projektion "PredP". Die theoretische Motivation für Bowers ist, damit eine einheitliche Struktur für Prädikation anzusetzen, die sowohl die Prädikation in Sätzen als auch die Prädikation in verbfreien Prädikationsstrukturen ('small clauses') abdeckt. Nach Chomsky wird durch diese Projektion die spezielle semantische Beziehung zwischen dem Subjekt auf der einen Seite und dem Verb mit seinen Objekten auf der anderen Seite ausgedrückt. Wir übernehmen die Annahme dieser speziellen Projektion. Das Verb bewegt sich in die Kopf-Position dieser Projektion, um die thematische Lizenz des Subjekts durch Pred' zu ermöglichen (und um die Thetalizenz an das ranghöchste Objekt zu erteilen). ${ }^{3}$

Aus Kasusgründen wird das Subjekt von der Spec-Position von PredP wegbewegt in die Spec-Position von IP. 


\section{Adverbiale der Art und Weise}

Es gibt eine ganze Reihe von Hinweisen darauf, daß Adverbiale der Art und Weise im deutschen Mittelfeld in einer zum Verb bzw. zum Prädikatskomplex ${ }^{4}$ adjazenten Stelle ihre Grundposition haben. Wir möchten dies hier anhand der Stellung des w-Indefinitums illustrieren. Indefinit interpretierte w-Pronomina sind stellungsfest, d.h. sie befinden sich stets in ihrer Grundposition und können nicht gescrambelt sein. Sie sind daher geeignet, Grundpositionen zu ermitteln:

(7) a. weil ein Professor wen beleidigt hat

b. *weil wen ein Professor beleidigt hat

c. weil Hans neulich wen beleidigt hat

d. *weil Hans wen neulich beleidigt hat

Mit diesem Test läßt sich zeigen, daß die Grundposition von Adverbialen der Art und Weise nach den Objekten positioniert ist:

(8) weil Maria heute was sorgfältig durchgearbeitet hat

Das nicht-umstellbare Indefinitpronomen mit Objektfunktion geht dem Adverbial der Art und Weise voran. Dies ist nur möglich, wenn das Adverbial der Art und Weise nach dem Objekt basisgeneriert wird.

Betrachten wir nun die möglichen Grundpositionen für ein Adverbial der Art und Weise (A\&W-Adverbial) im Englischen:

(9) (*Elegantly) Superstring theory (*elegantly) will (*elegantly) have (elegantly) accounted (elegantly) for these phenomena (elegantly)

Auf den ersten Blick hat man den Eindruck, daß sich hier eine andere Regularität manifestiert als im Deutschen. Das Englische scheint nach (9) mehr Optionen für ein Adverbial der Art und Weise zu bieten.

Einige Autoren gehen davon aus, daß A\&W-Adverbiale in satzfinaler Position basisgeneriert werden (s. z.B. Alexiadou 1997). Alexiadou argumentiert dafür, daß die Position des A\&W-Adverbials vor dem Vollverb auf Bewegung von "leichten" Elementen zurückzuführen sei. Da sie jedoch eine bestimmte funktionale Projektion ("VoiceP") als Landeplatz angibt, kann sie damit die Position zwischen Objekten oder zwischen Verb und PP nicht erfassen.

Wir wollen nun untersuchen, wie weit die Annahme trägt, daß die Bedingung (2(v)) auch für das Englische gilt. Betrachten wir hierzu die folgende Struktur:

(10) John has $\left[\operatorname{spoken}_{1}\right.$ [to his mother $\left[\mathrm{t}_{1}{ }^{\prime}\left[\right.\right.$ about the book $\left[\mathrm{t}_{1}\right.$ nicely $\left.\left.\left.]\right]\right]\right]$ In (10) ist die Verbkette angegeben. Das Adverbial am Satzende sei an die Grundposition des Verbs adjungiert. Das Verb, respektive seine Spur, wird minimal von dem Adverbial k-kommandiert. Wir nehmen an, daß in einer

Da für die Überlegungen im vorliegenden Artikel eine mögliche Aufspaltung der traditionellen funktionalen I ${ }^{0}$-Kategorie in $\mathrm{T}^{0}$ und $\mathrm{AgrS}{ }^{0}$ nicht relevant ist, nennen wir weiterhin die Projektion, in deren SpecPosition sich das englische Subjekt auf der Oberfläche befindet, die I-Projektion.

Zum Prädikatskomplex gehören bestimmte verbadjazente Konstituenten wie z.B. Direktionaladverbiale, Resultativprädikate und nicht-verbale Teile von Funktionsverbgefügen. Diese Elemente lassen sich im Mittelfeld nicht scrambeln und tragen zur Valenz des Prädikats bei. 
basisgenerierten Adjunktionsstruktur die K-Kommando-Domäne des adjungierten Knotens vom dominierenden Knoten beschränkt wird. Die K-Kommando-Domäne des adjungierten Knotens ist somit beschränkt auf die Adjunktionsstruktur. Der Kopf hingegen wird in seiner K-Kommando-Domäne durch den dominierenden Kopf einer Adjunktionsstruktur nicht beschränkt. ${ }^{5}$

Das Adverbial in (10) erfüllt die Bedingung für ein A\&W-Adverbial. Die Struktur in (10) erlaubt weitere Positionierungen des Adverbials unter Beachtung der Bedingung (2(v)). Diese ergeben sich durch Adjunktion an die anderen Glieder der Bewegungskette des Verbs:

$$
\text { John has }\left[\left[\operatorname{spoken}_{1} \text { nicely }\right]\left[\text { to his mother }\left[\left[\mathrm{t}_{1}{ }^{\prime} \text { nicely }\right] \text { [about the book }\left[\mathrm{t}_{1} \text { nicely }\right]\right]\right]\right]
$$

Die Adjunktion erfolgt jeweils nach rechts, da sich das Adverbial im Wirkungsbereich des Verbs befinden muß. Dessen Lizenzierungsbereich ist nach rechts. 6

Nun könnte man vermuten, daß die Position zwischen Verb und PP bzw. zwischen den beiden PPs dadurch zustandekommt, daß die PPs nach rechts extraponiert worden sind ("heavy constituent shift") und daß das Adverbial der Art und Weise stets an der gleichen Position steht. Es läßt sich jedoch zeigen, daß in (11) alle Argumente in ihren Grundpositionen stehen. Man betrachte hierzu den folgenden Unterschied:

(12) a. Bill looked carefully at the pictures of Miró

b. Bill looked yesterday at the pictures of Miró

c. Which painter did Bill look carefully at the pictures of?

d. *Which painter did Bill look yesterday at the pictures of?

Wie Costa (1996) beobachtet, ist eine Extraktion aus dem Präpositionalobjekt in (12c) möglich, jedoch nicht in (12d). Es ist ein wohl etabliertes Prinzip, daß aus einer A-quer-bewegten Konstituente nichts herausbewegt werden kann. Eine Möglichkeit, die Daten zu interpretieren, wäre, daß das Objekt in (12b) extraponiert wurde. (12b) liegt dann der folgende Satz zugrunde:

(13) Bill looked at the pictures of Miró yesterday

Die Grammatikalität von (12c) hingegen zeigt, das sich hier das Objekt in seiner Grundposition befindet. Die Daten in (12) entsprechen somit dem in (2) festgestellten Sachverhalt, daß ein Temporaladverbial anderen strukturellen Bedingungen zu genügen hat als ein A\&W-Adverbial. Ein Temporaladverbial kann nicht an V adjungiert werden.

Es läßt sich nun für die PPs in (11) entsprechend zeigen, daß sie jeweils in ihren Grundpositionen stehen:

(14) a. Who has John spoken nicely to about this book?

b. What has John spoken to his mother nicely about?

Die Position des Adverbials der Art und Weise zwischen den Objekten wie in (15) zeigt, daß die traditionelle Annahme, daß A\&W-Adverbiale an die V-Projektion adjungiert seien, die alle Objekte umfaßt, nicht haltbar ist.

$5 \quad$ Wir übernehmen die Definition von K-Kommando (Chomsky 1981), wonach ein Kopf sämtliche Phrasen 6 innerhalb seiner maximalen Projektion k-kommandiert.

Bislang nicht befriedigend geklärt werden konnte die Tatsache, daß zwischen Verb und einem NP-Objekt keine Adverbiale irgendeiner Art und somit auch keine Adverbiale der Art und Weise stehen können. Die Standardannahme, daß Kasuszuweisung im Englischen nur an eine adjazente Konstituente möglich ist, ist aus verschiedenen Gründen nicht überzeugend. Wir müssen diese Annahme aber übernehmen. 
(15) John has spoken to his mother nicely about the book

Da, wie (14b) zeigt, die about-PP nicht extraponiert ist, kann das Adverbial der Art und Weise nicht an die alle Objekte dominierende VP adjungiert sein. Ist, wie nach unserer Annahme, das A\&W-Adverbial an das Verb bzw. an eine Verbspur adjungiert, sind hingegen Daten wie in (15) erwartet. Man beachte wiederum, daß in der Position zwischen zwei Objekten tatsächlich nur A\&W-Adverbiale möglich sind, wenn kein 'heavy constituent shift' vorliegt:

(16) a. *They reduced the village inadvertently to ruins

b. *John spoke willingly to her

Auch dies ist erwartet. Adverbiale der Subjekthaltung wie inadvertently oder willingly, die die Einstellung des Subjektsreferenten zur Handlung beinhalten, können nicht an V adjungiert werden. Sie sind, worauf im nächsten Abschnitt eingegangen wird, ereignisinterne Adverbiale und werden nach (2(iv)) minimal von ihrem Bezugselement k-kommandiert. Sie müssen daher das Objekt k-kommandieren.

Man beachte, daß sich mit unseren Überlegungen auch unmittelbar ergibt, daß ein A\&W-Adverbial nicht vom Hauptverb entfernt positioniert werden kann:

(17) Superstring theory (*elegantly) will (*elegantly) have accounted for these phenomena

In (17) steht das Adverb zwar in potentiellen Adverbpositionen. Diese Positionen sind aber nur möglich für Adverbien, die einer anderen Klasse angehören, z.B. der Klasse der ereignisbezogenen Adverbien:

(18) a. We (now) have (now) been sitting here for three hours

b. George (already) has (already) been making plans

Ein A\&W-Adverbial ist in diesen Positionen nicht möglich, da es hier nicht an das Hauptverb adjungiert sein kann.

Sämtliche in (11) angegebenen möglichen Positionierungen des Adverbials ergeben dieselbe Interpretation eines Satzes. Es gibt aber noch eine weitere mögliche Stellung des A\&W-Adverbials. Es handelt sich um die Position vor dem Verb:

(19) John has been cleverly talking about the problem

Wichtig ist, daß diese Position zu einer anderen Interpretation führt als die bislang betrachteten Positionen nach dem Hauptverb. Cinque (1997) beobachtet, daß die Adverbialposition vor dem Verb und jene am Satzende simultan besetzt werden können:

(20) John has been cleverly talking about the problem stupidly

(20) ist nicht widersprüchlich, obwohl das erste Adverbial nicht als (subjektorientiertes) Satzadverbial interpretiert wird. Auch im Deutschen ist eine derartige Konstruktion möglich:

(21) Hans hat geschickt alle Fragen dumm beantwortet

Entsprechendes zu (20) ist nicht möglich mit der Position nach dem Verb: 
(22) *John has been talking cleverly about the problem stupidly

Entscheidend ist nun, daß nicht alle Adverbiale der Art und Weise in dieser präverbalen Position auftreten können:

(23) a. He will dance with Mary marvellously

b. *He will marvellously dance with Mary

c. She has shot at Paul badly

d. *She has badly shot at Paul

e. She has played the violin beautifully

f. *She has beautifully played the violin

g. John learned French perfectly

h. *John perfectly learned French

Bartsch (1972: 150f.) macht eine Unterscheidung bei den A\&W-Adverbialen, die für diese Beobachtungen relevant ist. Sie untersucht, ob ein Satz der Form 'Subjekt X A\&W-Adv' eine Paraphrase der Form 'Subjekt X, wobei Subjekt A\&W-Adv ist' ermöglicht.

Zur ersten Gruppe, die eine derartige Paraphrase erlaubt, gehört z.B. sorgfältig:

(24) a. Petra kocht sorgfältig

b. Petra kocht, wobei sie sorgfältig ist

Wunderbar gehört zur zweiten Gruppe und erlaubt diese Paraphrase nicht:

(25) a. Petra tanzt wunderbar

b. Petra tanzt, wobei sie wunderbar ist

(25a) und (b) können nicht dasselbe bedeuten. Wichtig ist nun, daß alle Adverbiale in (23) diese Paraphrase nicht erlauben.

Adverbiale der ersten Gruppe können dazu benutzt werden, den Subjektreferenten beim Vollzug der Handlung zu charakterisieren, bei Adverbialen der zweiten Gruppe ist dies nicht möglich. In dieser speziellen Verwendung sind die Adverbiale der ersten Gruppe aber keine Adverbiale der Art und Weise mehr. Sie charakterisieren hier, ähnlich wie Adverbiale der Subjekthaltung, den Subjektreferenten bezüglich des restlichen Satzes. Sie charakterisieren ihn aber nicht wie die Adverbiale der Subjekthaltung hinsichtlich einer mentalen Einstellung zur Handlung, sondern hinsichtlich des Handlungsvollzugs. Diese den Handlungsvollzug charakterisierenden Adverbiale sind offensichtlich keine Prädikate über den Prozeß mehr, wie dies für Adverbiale der Art und Weise gilt. In der Verwendung vor dem Verb gehören die Adverbiale der ersten Gruppe daher zu derselben Klasse wie die Adverbiale der Subjekthaltung, sie sind hier sogenannte ereignisinterne Adverbiale. Sie beziehen sich ebenfalls auf das Verhältnis ihres Bezugselementes zum Rest des Satzes. Für diese Klasse ist aber (2(iv)) einschlägig. Wie im nächsten Abschnitt gezeigt wird, ist für diese Klasse eine Position wie die des Adverbials in (19) charakteristisch.

Auf einen anderen Interpretationsunterschied zwischen der Positionierung vor und nach dem Hauptverb wird in Thomason/Stalnaker (1973) hingewiesen:

(26) a. He has been testing some bulbs slowly

b. He has been slowly testing some bulbs 
In (26a) wird jede einzelne Testhandlung als langsam charakterisiert, in (26b) wird hingegen gesagt, daß das gesamte Testen langsam durchgeführt wurde, die einzelnen Tests können dabei durchaus schnell vollzogen worden sein, mit Pausen dazwischen. Wenn slowly in der Position vor dem Verb steht, wird das gesamte Ereignis charakterisiert. Dieser Interpretationsunterschied zeigt, daß das Vorkommen des Adverbials in (26b) nicht als A\&W-Adverbial $\mathrm{zu}$ analysieren ist, sondern als ereignisbezogenes Adverbial. Auf die Positionierungsoptionen ereignisbezogener Adverbiale wird in Abschnitt 5 eingegangen.

\section{Adverbiale der Subjekthaltung}

Wir haben eingangs die Erwartung formuliert, daß sich Unterschiede im Verhalten der Adverbiale im Deutschen und Englischen nicht aufgrund unterschiedlicher Eigenschaften der Adverbiale ergeben, sondern daß diese vielmehr ein Resultat der unterschiedlichen Satzstrukturen in den beiden Sprachen sind. Wir wollen unter diesem Blickwinkel den folgenden Unterschied in der "Orientierung" eines Adverbials diskutieren:

(27) a. that Joan willingly was examined by the doctor (willingly bezieht sich auf Joan)

b. daß Otto bereitwillig von dem Doktor untersucht wurde (bereitwillig bezieht sich auf den Doktor)

Im Prinzip kann das Adverbial natürlich auch im Englischen auf das Agens bezogen werden. Dazu ist aber eine andere Positionierung nötig:

(28) that Joan was examined willingly by the doctor (willingly bezieht sich auf the doctor)

Interessant ist der Kontrast der Daten in (27). Willingly gehört zu den sogenannten "Adverbialen der Subjekthaltung" (Pittner 1996). Nach Frey/Pittner (i.E.) gehören diese zur Klasse der ereignisinternen Adverbiale. Ereignisinterne Adverbiale werden in ihrer Grundposition minimal von ihrem Bezugselement kkommandiert. Bei den Adverbialen der Subjekthaltung ist das Bezugselement das ranghöchste Argument der Argumentstruktur. Die Namensgebung für diese Adverbiale ist etwas irreführend. Zwar ist das ranghöchste Argument häufig das Subjekt, aber nicht notwendigerweise. Auch bei anderen ranghöchsten Argumenten ist ein derartiges Adverbial möglich:

(29) weil dem Peter versehentlich eine Tasse hinunter gefallen ist

In den Beispielen in (27) und (28) liegt ein Passiv vor. Das externe Argument des Aktivs kann in der passivischen Verbform unrealisiert bleiben oder durch eine von/by-Phrase realisiert werden. Die von/by-Phrase zeigt Eigenschaften eines Adjunkts. Wichtig ist, daß in beiden Fällen, also auch bei Nicht-Präsenz der PP, dieses Argument syntaktisch aktiv ist. Dies kann z.B. durch Bindungs- oder Kontrollphänomene gezeigt werden (s. z.B. Roberts 1987, Frey 1993):

(30) a. Briefe wurden einander geschrieben

b. Das Schiff wurde versenkt, um die Versicherungsprämie zu kassieren

Man spricht daher davon, daß beim Passiv das externe Argument des entsprechenden Aktivs implizit bleibt. Dies bedeutet, daß es stets in der syntaktischen Struktur präsent ist, obwohl es gar nicht oder nur durch ein Adjunkt 
realisiert wird. Als syntaktischer Repräsentant des impliziten Arguments wird z.B. von Roberts (1987) oder Frey (1993) die passivische Verbmorphologie bzw. das passivische Verb angenommen. Es ist dieser syntaktische Repräsentant, der eine Erklärung von Daten wie in (30) gestattet.

Welches ist das ranghöchste Argument eines passivischen Verbs? Das ranghöchste Argument eines Prädikats ist jenes Argument, für das gilt, daß es kein anderes Argument des Prädikats mit einer größeren KKommando-Domäne gibt. Dieses entspricht bei einem Prädikat, welches kein Raising-Prädikat ist, jener Argumentstelle, die in der konzeptuellen Struktur des Verbs den höchsten Hierarchieplatz einnimmt (s. z.B. Bierwisch 1988, Jackendoff 1990a) und die syntaktisch repräsentiert ist. Das externe Argument des Aktivs ist somit auch das ranghöchste Argument des korrespondierenden Passivverbs. Es ist nach wie vor das prominenteste Argument in der konzeptuellen Struktur (in der Regel ein Agens), es ist syntaktisch repräsentiert und sein Repräsentant k-kommandiert alle anderen Argumente. Das implizite Argument ist somit das ranghöchste Argument eines passivischen Verbs.

Kehren wir nun zurück zu den Daten in (27) und (28). Dieser Unterschied zwischen Deutsch und Englisch kann mit der, unabhängig motivierten, unterschiedlichen strukturellen Position des Subjekts in den beiden Sprachen in Beziehung gesetzt werden. Die Oberflächenposition des Subjekts befindet sich im Englischen außerhalb des Rektionsbereichs des Hauptverbs. Für das Deutsche hingegen gibt es eine Reihe von Gründen, anzunehmen, daß das Subjekt auf der Oberfläche innerhalb des Rektionsbereichs des Verbs positioniert ist (Haider 1993). Die bekannteste Evidenz für diesen Unterschied bezieht sich auf Extraktion:

(31) a. *Who ${ }_{1}$ would [to play chess with $t_{1}$ ] have pleased you?

b. Mit wem ${ }_{1}$ würde $\left[\mathrm{t}_{1}\right.$ Schach spielen zu dürfen] dich sehr freuen?

Eine klassische Bedingung für Extraktion besagt, daß aus einer Phrase, welche nicht lexikalisch, d.h. durch ein Element einer der vier Hauptkategorien, regiert wird, keine Konstituente wegbewegt werden kann. ${ }^{7}$ Der Subjektsatz in (31a) befindet sich in der Spec-Position einer funktionalen Projektion und wird, da außerhalb der PredP, nicht vom Hauptverb lexikalisch regiert. Deshalb führt die Extraktion einer Phrase aus dem Subjektsatz zur Ungrammatikalität. Die Grammatikalität von (31b) spricht dann dafür, daß der Subjektsatz im Deutschen lexikalisch vom Hauptverb regiert wird. Das Subjekt befindet sich somit innerhalb des Rektionsbereichs des Verbs.

Aus dieser Überlegung folgt, daß sich das Adverbial in (27a) nicht im Rektionsbereich des Hauptverbs befindet, sondern lediglich im Bereich des Auxiliars, welches in einer funktionalen Position steht (in der I-Position). Das Auxiliar hat zwar keine Theta-Struktur, aber es hat eine theta-freie Subjektstelle. Dies ist sein ranghöchstes Argument, denn das implizite Argument des Passivverbs ist in der Domäne des Auxiliars nicht präsent. Auf dieses Subjekt ist das Adverbial bezogen, da es von diesem ranghöchsten Argument minimal kkommandiert wird. Das Subjekt der Passivkonstruktion ist das logische Objekt des Hauptverbs und wir erhalten die fragliche Interpretation von (27a).

Die Situation ist eine andere bei (27b). Das ganze deutsche Mittelfeld gehört zum Rektionsbereich des 
Frey/Pittner - 10 -

Hauptverbs. Das Subjekt befindet sich daher innerhalb dieses Bereichs und somit auch das Adverbial. Innerhalb dieser Domäne ist jedoch das implizite Argument des Passivs das ranghöchste Argument. Das Hauptverb bildet mit werden einen Prädikatskomplex. Der Prädikatskomplex k-kommandiert das gesamte Mittelfeld und damit auch das Adverbial. Das Adverbial wird daher auf das implizite Argument bezogen, da dies das prominenteste Argument in jener Domäne ist, in der sich das Adverbial befindet. Das Gleiche gilt für das englische Beispiel (28). Das Adverbial steht in diesem Beispiel innerhalb der VP des Hauptverbs und wird von diesem kkommandiert. Wiederum ist das implizite Argument das prominenteste Argument in dieser Domäne. Dies ergibt die Interpretation von (28).

Der folgende Satz schließlich ist ambig:

(32) that Joan was willingly examined by the doctor

Im Passivsatz bewegt sich das Verb nicht in die Kopfposition der PredP, da es in dessen Spec-Position kein externes Argument gibt, das thetamarkiert würde. (32) erlaubt verschiedene Strukturzuweisungen. Es gibt eine Analyse, bei der willingly an die V-Projektion von examined adjungiert ist. Bei dieser Analyse wird das Adverb auf das implizite Argument bezogen, d.h. im vorliegenden Beispiel auf the doctor. Es gibt eine zweite Analyse, bei der willingly höher adjungiert ist, und zwar an die V-Projektion, die von der Grundposition des Auxiliars aufgespannt wird. ${ }^{8}$ Unter dieser Analyse ist das Oberflächensubjekt das minimal k-kommandierende Bezugselement, und es resultiert die Lesart, bei der willingly auf Joan bezogen wird.

Die Daten in (27), (28) und (32) machen deutlich, daß Adverbiale der Subjekthaltung eine Sensitivität für die Oberflächenverhältnisse des Satzes aufweisen. Dies zeigt sich auch in anderen Beispielen. Im Deutschen müssen z.B. Adverbiale der Subjekthaltung im Mittelfeld vom ranghöchsten Argument auf der Oberfläche kkommandiert werden:

(33) a. weil ein Kollege freiwillig diese Arbeit übernommen hat

b. *weil freiwillig ein Kollege diese Arbeit übernommen hat

Es genügt nicht, daß die Grundposition des Adverbials vom Bezugselement k-kommandiert wird. Diese Oberflächensensitivität zeigt sich auch im englischen Aktivsatz:

(34) a. Terry intentionally has been reading Hamlet

b. Terry has intentionally been reading Hamlet

c. Terry has been intentionally reading Hamlet

d. Terry has been reading Hamlet intentionally

Die Beispiele zeigen, daß ein Adverbial der Subjekthaltung von der oberflächenstrukturellen A-Position des Bezugselements minimal k-kommandiert werden muß. ${ }^{9}$ Es muß offensichtlich nicht der Fall sein, daß das Bezugselement in seiner Grundposition das Adverbial k-kommandiert. Diese Orientierung an der Oberfläche ist eine Besonderheit der Adverbiale der Subjekthaltung. Für sie gilt die Bedingung (2(iv)) als eine Oberflächenbedingung. Für die anderen ereignisinternen Adverbiale gilt, daß (2(iv)) bezüglich der 
Grundpositionen zu erfüllen ist. Dies wird im nächsten Abschnitt für die Lokaladverbiale illustriert.

\section{Temporal- und Lokaladverbiale}

In diesem Abschnitt werden wir zunächst kurz auf die Grundpositionen von Temporal- und Lokaladverbialen im Deutschen eingehen, um dann zu überprüfen, ob im Englischen die gleichen Regularitäten auftreten.

Einer Standardannahme zufolge ist die Grundposition des Lokal- sowie des Temporaladverbials im deutschen Mittelfeld unterhalb des Subjekts (z.B. Grundzüge 1981, Abraham 1986). ${ }^{10}$ Es gibt jedoch einige Evidenz dafür, daß die Grundposition des Temporaladverbials, im Gegensatz zu der des Lokaladverbials, oberhalb des ranghöchsten Arguments anzusiedeln ist.

Die folgenden Daten zeigen, daß das Temporaladverbial eine andere Grundposition hat als das Lokaladverbial:

(35) a. ??weil wer morgen den Balken abstützen sollte

b. weil wer hier die Leitung reparieren sollte

c. ??weil wo jemand etwas reparieren soll

d. weil wer mit der Leiter den Balken abstützen sollte

Die betrachteten Adverbialtypen verhalten sich bezüglich des Subjekts unterschiedlich. W-Indefinita sind stellungsfest. Das Temporaladverbial hat somit nach (35a) seine Grundposition oberhalb der Grundposition des ranghöchsten Arguments, ein Lokaladverbial hat, wie (35b) und (c) zeigen, seine Grundposition unterhalb des ranghöchsten Arguments. Das Lokaladverbial in (35b) verhält sich in dieser Hinsicht wie das Instrumentaladverbial in (d), bei dem davon ausgegangen werden kann, daß es seine Grundposition unterhalb des ranghöchsten Arguments einnimmt (für eine ausführlichere Erörterung und die Anwendung weiterer Tests s. Frey/Pittner i.E., 4.3).

Wir haben die Grundposition des Temporaladverbials oberhalb des ranghöchsten Arguments dahingehend gedeutet, daß Temporaladverbiale ein Ereignis als Ganzes situieren, mithin ereignisbezogene Adverbiale sind, während Lokaladverbiale eher einzelne Beteiligte lokalisieren, in diesem Sinn über ein Bezugsargument verfügen und sich daher wie ereignisinterne Adverbiale verhalten.

Betrachten wir nun einige englische Daten. Hierfür wird Prinzip C der Bindungstheorie wichtig sein. Prinzip C können wir wie folgt formulieren:

(36) Ein R-Ausdruck $\alpha$ darf nicht mit einem Ausdruck $\beta$ koindiziert sein, wenn eine der folgenden Bedingungen auf der S-Struktur gilt:

(i) $\quad \beta$ k-kommandiert $\gamma$, oder

(ii) $\quad \beta$ k-kommandiert eine Spur von $\gamma$

wobei $\gamma=\alpha$ oder $\gamma$ enthält $\alpha$

Damit ergeben sich z.B. die folgenden Daten des Deutschen:

10 Hier ist die Rede von den kanonischen Lokal- und Temporaladverbialen, die ein Ereignis räumlich oder zeitlich situieren, nicht jedoch von den Frameadverbialen, die den Geltungsbereich einer Proposition räumlich oder zeitlich einschränken (s. dazu Maienborn 1996). 
(37) a. weil Marias Freund heute Maria ${ }_{1}$ zum Flughafen bringt

b. *weil ich Peter ${ }_{1}$ mit einem alten Ârtikel von Peter ${ }_{1}$ konfrontierte

c. *Mit einem alten Artikel von Peter ${ }_{1}$ habe ich Peter $_{1}$ konfrontiert

In (37a) ist Prinzip C erfüllt. Kein Vorkommen des R-Ausdrucks k-kommandiert das andere Vorkommen. In (37b) ist (36(i)) verletzt. Das Akkusativobjekt k-kommandiert das Präpositionalobjekt (und damit alle Elemente, die darin vorkommen). In (37c) ist (36(ii)) verletzt. Die Grundposition des Präpositionalobjekts im Vorfeld befindet sich im K-Kommando-Bereich des Akkusativobjekts. Daher k-kommandiert das direkte Objekt die Spur der Phrase im Vorfeld.

Diverse Adverbialtypen können im Englischen an der Spitze des Satzes auftreten. Diese zeigen nun Unterschiede im Hinblick auf Prinzip-C-Effekte. In Speas (1990) finden sich die folgenden Daten:

(38) a. On Rosa ${ }_{1}$ 's birthday she ${ }_{1}$ took it easy

b. *On Rosa 1 's lawn she 1 took it easy

c. *With $\mathrm{John}_{1}$ 's computer he ${ }_{1}$ began to write a book of poetry

d. In Ben 1 's office he ${ }_{1}$ is an absolute dictator

e. *In Ben ${ }_{1}$ 's office he 1 lay on his desk

f. For Mary ${ }_{1}$ 's valor she ${ }_{1}$ was awarded a purple heart

g. *For Mary ${ }_{1}$ 's brother she 1 was given some old clothes

Eine genaue strukturelle Analyse des Bereichs vor dem englischen Subjekt ist für unsere Zwecke nicht wichtig. ${ }^{11}$ Entscheidend ist, daß ein Element dort basisgeneriert sein kann und in diesem Fall vom Subjekt nicht k-kommandiert wird. Ein Element in diesem Bereich kann aber auch durch Bewegung dorthin gelangt sein. Dies gilt z.B. für topikalisierte Argumente.

Nehmen wir nun an, daß die verschiedenen Adverbialklassen des Englischen auch den Bedingungen in (2) unterliegen. Dann ergeben sich die folgenden Erklärungen der Daten in (38). Ein Temporaladverbial gehört zur Klasse der ereignisbezogenen Adverbiale. Die Bedingung (2(iii)) für die Positionierung eines Temporaladverbials ist in (38a) erfüllt, wenn das Adverbial in in seiner Oberflächenposition basisgeneriert ist. Daher ergibt sich keine Prinzip-C-Verletzung in (38a). In (38b) tritt ein Lokaladverbial auf. Für ein solches Adverbial ist Bedingung (2(iv)) einschlägig. Das Bezugsargument für ein Lokaladverbial ist das ranghöchste Argument. Daher muß es im K-Kommando-Bereich (der Grundposition) des Subjekts basisgeneriert worden sein. Das Lokaladverbial muß also in (38b) seine Oberflächenposition durch eine Derivation erreicht haben. Somit befindet sich in (38b) eine Spur der vorangestellten Phrase im K-Kommando-Bereich des Subjekts und es ergibt sich mit (36(ii)) eine Prinzip-C-Verletzung. Analog ist die Situation in (38c). Nach Frey/Pittner (i.E.) gehören Instrumentaladverbiale bezüglich (2) zur gleichen Klasse wie Lokaladverbiale. Auch für sie ist somit Bedingung (2(iv)) einschlägig. Deshalb befindet sich auch in (38c) eine Spur der vorangestellten Phrase im KKommando-Bereich des Subjekts und es resultiert ebenfalls eine Prinzip-C-Verletzung. (38d) und (38e) dienen zum Vergleich. In (38d) tritt ein Frame-Adverbial auf. Die Zuweisung einer permanenten Eigenschaft wird hier durch dieses Adverbial auf einen bestimmten Ort eingeschränkt. Für ein Frame-Adverbial ist (2(i)) zu erfüllen.

11 Rizzi (1995) nimmt an, daß sich oberhalb TP (= IP in der traditionellen Analyse) eine Reihe funktionaler Projektionen entfalten. Eine davon ist Top ${ }^{0}$, in deren Spezifikator topikalisierte Elemente positioniert werden. Die traditionelle Analyse adjungiert topikalisierte Konstituenten an IP. 
Frey/Pittner - 13 -

Das Frame-Adverbial in (38d) kann somit in seiner Oberflächenposition basisgeneriert werden und es ergibt sich wie in (38a) keine Prinzip-C-Verletzung. Dieselbe Phrase tritt in (38e) als normales Lokaladverbial auf. Für dieses Beispiel ist demnach die gleiche Überlegung wie für (38b) einschlägig. Betrachten wir schließlich die Beispiele (f) und (g). In (f) tritt satzinitial ein Adverbial des Grundes auf. Für ein Adverbial des Grundes ist nach Frey/Pittner (i.E.) Bedingung (2(iii)) zu beachten. Wenden wir diese Bedingung auf (f) an, so erlaubt sie uns, das Adverbial als in seiner Oberflächenposition basisgeneriert zu analysieren. Wir erwarten daher keine Prinzip-CVerletzung. In (g) schließlich tritt dieselbe Phrase als Benefaktiv-Phrase auf. Die Grundposition einer Benefaktiv-Phrase befindet sich im K-Kommandobereich des Subjekts. Wir erwarten daher eine Prinzip-CVerletzung für $(38 \mathrm{~g})$.

Für die soeben angestellten Überlegungen ist entscheidend, daß nach (2) die Adverbialphrasen in (38 a,d,f) in ihrer Oberflächenposition basisgeneriert werden können, während die Adverbiale in $(38$ b,c,e,g) ihre Oberflächenposition durch Bewegung erreichen, so daß sich hier eine Spur im K-Kommandobereich des Subjekts befindet. Es ist möglich, für diesen Unterschied weitere Evidenz zu finden. Bislang gibt es zwar noch wenige Testmöglichkeiten, um festzustellen, ob eine Phrase vor dem Subjekt im Englischen basisgeneriert oder bewegt ist. Es gibt aber ein Phänomen, welches die gewünschte weitere Evidenz liefern kann.

Im Englischen ist bekanntlich eine Subjektspur nach einer Konjunktion wie that normalerweise nicht möglich:

(39) a. *I asked what ${ }_{1}$ Leslie said that $t_{1}$ had made Robin give a book to Lee

b. *This is the tree which $_{1}$ I said that $\mathrm{t}_{1}$ had resisted my shovel

Es gibt aber Adverbiale, die, wenn direkt nach that positioniert, den 'that-trace'-Effekt aufheben:

(40) a. I asked what ${ }_{1}$ Leslie said that in her opinion $t_{1}$ had made Robin give a book to Lee

b. This is the tree which $_{1}$ I said that just yesterday $t_{1}$ had resisted my shovel

Browning (1996) beobachtet nun, und dies ist entscheidend für unsere Überlegungen, den Unterschied von (40) zu den folgenden Daten:

(41) a. *Who did Leslie think that, this present, Kim gave to?

b. *Who did Robin say that, this present, gave Lee?

Die Daten in (41) zeigen, daß eingebettete Topikalisierung eines Arguments generell die Extraktion eines anderen Elements blockiert und damit insbesondere nicht zur Aufhebung des 'that-trace'-Effekts führt (41b).

Für Brownings Erklärung des Unterschieds zwischen (40) und (41) ist entscheidend, daß sie annimmt, daß die topikalisierten Argumente in (41) ihre Oberflächenposition durch Bewegung erreichen, wohingegen sie annimmt, daß die Adverbiale von (40) in ihrer Oberflächenposition basisgeneriert sind.

In Browning (1996) wird nicht zwischen verschiedenen Adverbialtypen unterschieden. Es werden Beispiele mit Bereichsadverbialen, Satzadverbialen und Temporaladverbialen gegeben, welche alle zur Aufhebung des 'that-trace'-Effekts führen. Wenn wir nun aber systematisch die Adverbiale von (38) auf ihre Fähigkeit zur Aufhebung des 'that-trace'-Effekts überprüfen, so finden wir die folgenden Daten: 
(42) a. Who ${ }_{1}$ do you think that on Rosa's birthday $t_{1}$ took it easy

b. ${ }^{*} \mathrm{Who}_{1}$ do you think that on Rosa's lawn $\mathrm{t}_{1}$ took it easy

c. $* W_{1} o_{1}$ do you think that with John's computer $t_{1}$ began to write a book of poetry

d. Who $_{1}$ do you think that in Ben's office $t_{1}$ is an absolute dictator

e. ${ }^{*} \mathrm{Who}_{1}$ do you think that in Ben's office $\mathrm{t}_{1}$ lay on his desk

f. Who ${ }_{1}$ do you think that for this reason $t_{1}$ was awarded a prize

g. $* \mathrm{Who}_{1}$ do you think that for Mary's brother $\mathrm{t}_{1}$ was given some old clothes

In (42a) tritt ein Temporaladverbial, in (42d) eine Frame-Adverbial und in (42f) ein Kausaladverbial auf. Somit führen genau jene Adverbialtypen, welche nach (2) in (42) in ihrer Oberflächenposition basisgeneriert werden können, zu einer Aufhebung des 'that-trace'-Effekts. Eine Aufhebung des 'that-trace'-Effekts durch eine Phrase zwischen dem Komplementierer und dem Subjekt ist nach Browning (1996) nur möglich, wenn diese Phrase in ihrer Basisposition steht. Genau jene Phrasen hingegen, welche ihre Position vor dem Subjekt durch Bewegung erreichen, können nach Browning den 'that-trace'-Effekt nicht aufheben. Dies sind in (42) genau jene Phrasen, die auch nach (2) bewegt worden sein müssen: Lokal- und Instrumentaladverbiale und Benefaktivphrasen.

Ebenso wie die Prinzip-C-Daten bestätigen also die Überlegungen von Browing die Regularitäten von (2), wonach z.B. satzinitiale Temporal-, Kausal- oder Frame-Adverbiale im Englischen in dieser Position basisgeneriert werden können, während dies z.B. für Lokal- und Instrumentaladverbiale nicht gilt.

Wir wollen nun noch eine weitere Beobachtung über Temporal- und Lokaladverbialen kommentieren. Temporaladverbiale können auch in dem Bereich zwischen Subjekt und Verb vorkommen. Dies ist zwar nicht generell der Fall, aber es gilt für die 'kleinen' Vertreter:

(43) a. He (then) will (then) have ((?)then) read the book

b. He (*tomorrow) will (*tomorow) have (*tomorow) read the book

Phrasen wie tomorrow oder yesterday können wohl als PPs angesehen werden, während dies für then nicht zutrifft. Wir können über den Unterschied in (43) keine Aussagen machen. Wir wollen aber auf den Unterschied zwischen (43a) und (44) hinweisen:

(44) She $(*$ here $)$ will $(*$ here $)$ be $(*$ here $)$ reading this book

Die Vorkommen der Temporaladverbiale in (43a) erfüllen die relevante Bedingung (2(iii)). Für ein Lokaladverbial wie in (44) hingegen ist Bedingung (2(iv)) bezüglich der Grundpositionen einschlägig. Danach muß die Grundposition eines Lokaladverbials von der Grundposition des Subjekts k-kommandiert werden. Dies ist in (44) für die beiden ersten angegebenen Positionen offensichtlich nicht der Fall. Die Grundposition des Subjekts befindet sich unterhalb des Auxiliars be. Aber auch die dritte in (44) angegebene Position ist nicht möglich. Die Grundposition des Subjekts befindet sich in der Spec-Position von Pred. Chomsky (1995) nimmt an, daß die Konfiguration Pred-VP (bei Chomsky ' $v$-VP') die kausative oder agentive Rolle des externen Arguments ausdrückt. Wir nehmen an, daß an Pred' nur jene Adverbiale adjungiert werden können, die diese Rolle weiter charakterisieren. Dies sind Adverbiale der Subjekthaltung und jene Vorkommen von ursprünglichen Adverbialen der Art und Weise, die nach Abschnitt 3 das Subjekt beim Handlungsvollzug charakterisieren. Lokaladverbiale charakterisieren nicht die kausative oder agentive Rolle. Sie können deshalb nicht an Pred' 
adjungiert werden. ${ }^{12}$

Zum Abschluß dieses Abschnitts wollen wir noch eine Bemerkung zu den Frequenzadverbialen machen. Wie im Deutschen haben auch im Englischen die Frequenzadverbiale viele Möglichkeiten der Positionierung. Dies spiegelt den semantischen Sachverhalt wider, daß Frequenzadverbiale sowohl Prozesse als auch Ereignisse charakterisieren können:

(45) Hans hat mehrmals jede Türe zweimal angestrichen Im Englischen können Frequenzadverbiale in den folgenden Positionen auftreten:

(46) (Frequently) she (frequently) has (frequently) been (frequently) talking (frequently) to Mary (frequently)

Da sie Prozeß- und Ereignismodifikatoren sind, erwarten wir diese Verteilung. Die Frequenzadverbiale vereinigen die Möglichkeiten der A\&W-Adverbiale und der Temporaladverbiale.

In Cinque (1997) werden ausführlich die folgenden Beispiele diskutiert:

(47) a. John intentionally knocked on the door twice

b. John twice knocked on the door intentionally

Das Interessante an diesen Beispielen ist, daß (47a) ambig ist und (47b) eindeutig. (47a) besitzt eine Lesart mit Skopus von intentionally über twice und eine Lesart mit Skopus von twice über intentionally. (47b) besitzt nur die letztere Lesart. Cinque sieht darin ein schlagendes Argument gegen die Möglichkeit von Rechtsadjunktion in der Grammatik. Cinque zufolge sollten unter dieser Option beide Sätze ambig sein. Er schlägt eine äußerst komplexe Ableitung der Sätze aus einer Struktur ohne Rechtsadjunktion vor. Die Ableitung benutzt Optionen, die bislang in der Grammatik nicht etabliert sind.

Wir werden in Abschnitt 7 unsere Alternative zur Rechtsadjunktion skizzieren. Wir wollen aber hier zunächst darauf hinweisen, daß in einem System, welches die Bedingungen (2) beinhaltet und welches Rechtsadjunktion zuläßt, die Interpretationsmöglichkeiten der Beispiele in (47) abgeleitet werden können.

In (47a) ist es möglich, twice als prozeßbezogenes oder als ereignisbezogenes Frequenzadverbial zu analysieren. Im ersten Fall ist es an die Verbspur adjungiert, im zweiten Fall ist PredP die kleinste Phrase, die für die Adjunktion in Frage kommt, da ereignisbezogene Adverbiale die Grundposition des Subjekts kkommandieren müssen. Das Adverbial der Subjekthaltung intentionally kann an Pred' adjungiert werden (s. Abschnitt 4). Das ereignisbezogene twice k-kommandiert diese Position, das prozeßbezogene twice wird von dieser Position k-kommandiert. Aufgrund der unterschiedlichen Analysemöglichkeiten für twice ergibt sich somit die Ambiguität von (47a).

Die Situation ist eine andere in (47b). Nach den Überlegungen des Abschnitts 3 kann twice in diesem

12 Der folgende Satz ist ebenfalls nicht grammatisch ohne 'heavy shift' des Präpositionalobjekts:

(i) *John will talk here with her

Adjunktion von here an die VP würde Bedingung (2(iv)) erfüllen. Die Adjunktion des Adverbials an die VP blockiert aber die Thetamarkierung des Präpositionalobjekts, welches sich in der Spec-Position der VP befindet, durch das Verb in Pred. 
Beispiel nur als ereignisbezogenes Adverbial interpretiert werden, da es sich vor der Pred-Position befindet. Eine prozeßbezogene Lesart würde eine Position nach dem Verb erfordern. Das Adverbial der Subjekthaltung intentionally ist an Pred' adjungiert. Da twice nur als ereignisbezogenes Adverbial analysiert werden kann, ergibt sich die Eindeutigkeit des Beispiels.

Wir können den Status eines weiteren Beispiels aus Cinque (1997) plausibilisieren.

(48) ??John intentionally twice knocked on the door

Obwohl es sich um mögliche Positionen für die Adverbiale handelt, ist der Satz nicht wohlgeformt. Das Frequenzadverbial muß auch in diesem Beispiel als ereignisbezogen analysiert werden. In (48) k-kommandiert aber intentionally das Frequenzadverb. Dies bedeutet, daß ein ereignisinternes Adverbial ein ereignisbezogenes Adverbial k-kommandiert. Dies verletzt die Bedingung (2(iii)).

\section{Satzadverbiale}

Satzadverbiale sind nach Auffassung einer ganzen Reihe von Autoren sensitiv für die Topik-KommentarGliederung eines Satzes: Sie stehen nach den Topik-Konstituenten. Wie im Deutschen scheint es auch im Englischen schwierig zu sein, eine Phrase, die nicht Topik sein kann, vor ein Satzadverbial zu stellen. Existentiell interpretierte nackte Plurale, die nicht topikfähig sind, da sie neue Diskursentitäten einführen, können im Deutschen nicht vor dem Satzadverbial auftreten.

(49) *weil Mädchen glücklicherweise geboren wurden

In Kiss (1996:129) wird beobachtet, daß auch im Englischen eine derartige Konstituente nicht vor einem Satzadverbial erscheinen kann:

(50) ??Boys luckily were born

Wenn man also die Position bezüglich des ranghöchsten Arguments ermitteln will, so muß man darauf achten, daß das ranghöchste Argument keine Topik-Konstituente ist. Dies ist auf jeden Fall dann gegeben, wenn das ranghöchste Argument auf nichts speziell referiert, da Topikalität an Referentialität als Voraussetzung gebunden ist.

(51) *weil wer vermutlich geraucht hat

(51) zeigt, daß Satzadverbiale ihre Grundposition oberhalb der Grundposition des ranghöchsten Arguments haben. Ebenso läßt sich zeigen, daß Satzadverbiale, da sie Propositionsbezug haben, höher liegen als alle ereignisbezogenen Adverbiale, also z.B. kausale Adverbiale:

(52) a. weil er wahrscheinlich wegen was eingeschnappt war

b. *weil er wegen was wahrscheinlich eingeschnappt war

Zur vollständigen Spezifikation eines Ereignisses gehört seine temporale Verankerung. Wenn nun Satzadverbiale über ein vollständig spezifiziertes Ereignis prädizieren, erwarten wir, daß sich in der Syntax eine 
Beziehung zwischen dem Satzadverbial und der temporalen Verankerung zeigt. Weil das finite Verb als der Repräsentant der temporalen Verankerung eines Ereignisses gelten kann, sollte das finite Verb im KKommandobereich des Satzadverbials auftreten müssen. Da dies im deutschen Mittelfeld wegen der Verbendstellung zwangsläufig gegeben ist, wird diese Bedingung im Deutschen nur bei gemeinsamer Topikalisierung des Satzadverbials mit dem infiniten Verb sichtbar. Betrachten wir daher die folgenden Daten:

(53) a. weil in diesem Land glücklicherweise viel gelacht wird

b. viel gelacht wird in diesem Land glücklicherweise

c. *Glücklicherweise viel gelacht wird in diesem Land

d. *Vermutlich geraucht wird heute abend

In (53a) und (b) ist das finite Verb bzw. seine Spur im K-Kommando-Bereich des Satzadverbials. Dies ist nicht der Fall in (c) und (d). Es ist keine plausible Annahme, daß sich im komplexen Vorfeld eine Spur des finiten Verbs befinden könnte (s. z.B. Haider 1993). Die Ungrammatikalität dieser Sätze können wir somit mit der Bedingung erfassen, daß Satzadverbiale das finite Verb (respektive eine Spur desselben) k-kommandieren müssen.

Betrachten wir nun die Verhältnisse im Englischen. Als erstes kann man feststellen, daß auch hier die Bedingung erfüllt sein muß, daß die Satzadverbiale die ereignisbezogenen Adverbiale k-kommandieren müssen. Dies erkennt man an folgendem Kontrast:

(54) a. George probably always will be singing that song

b. *George always probably will be singing that song

Betrachten wir als nächstes die möglichen Grundpositionen für Satzadverbiale:

(55) (Probably) She (probably) will (probably) have (*probably) answered the re porter's questions (*probably)

Für die ersten beiden Positionen am Beginn des Satzes ist klar, daß das Satzadverbial die Bedingung des KKommandos über das finite Verb erfüllt. Für die dritte mögliche Position ist dies nicht unmittelbar deutlich. Die Frage ist demnach, warum der folgende Satz grammatisch ist:

(56) She will probably have answered the reporter's questions

Es gibt Evidenz dafür, daß im Englischen finite Auxiliare und Modalverben in einer V-Position basisgeneriert und dann in die funktionale Finitheitsposition bewegt werden. Daß finite Auxiliare und Modalverben im Englischen eine andere strukturelle Position einnehmen als finite und infinite Hauptverben und infinite Auxiliare ist unstrittig. Dies zeigt sich z.B. bei Inversion und bei Negation:

(57) a. Had he left?

b. Must they leave?

c. *Leave they?

d. They had not left

e. They must not leave

f. *They left not

Man nimmt an, daß die finiten Auxiliare und die Modalverben in der funktionalen Projektion $\mathrm{I}^{0}$ stehen, während 
dies für die anderen Verben nicht gilt. Um den Zusammenhang der finiten Auxiliare zu den infiniten Formen zu bewahren, ist anzunehmen, daß alle Auxiliare in einer V-Position basisgeneriert werden. Die finiten Auxiliare werden dann nach $\mathrm{I}^{0}$ bewegt. Aber auch für Modalverben ist die Annahme, daß sie $\mathrm{I}^{0}$ durch Bewegung erreichen, sinnvoll. Dies erlaubt, davon auszugehen, daß alle verbalen Elemente des Englischen von der Kategorie V sind. Eine empirische Evidenz für die Bewegung der Modalverben ergibt sich durch Skopusdaten. Betrachten wir die folgenden Sätze aus Ernst (1991):

(58) a. George was obviously frequently associated with criminals

b. Gary can apparently lift 100 pounds

c. Gary already can clearly lift 100 pounds

(58a) dient lediglich zum Vergleich. Der Satz ist eindeutig. Er hat nur die Lesart, die der Oberflächenreihenfolge der Adverbiale entspricht. Die Sätze (56b) und (c) hingegen sind ambig. Sie haben jeweils eine Lesart, bei der das nach dem Modalverb stehende Adverbial Skopus nimmt über dieses Modalverb. (58b) hat z.B. die Lesart: Es ist offensichtlich, daß G. 100 Pfund heben kann. (58c) hat die Lesart: Es ist bereits der Fall, daß G. offensichtlich 100 Pfund heben kann. Interessanterweise kann in (58c) das Adverb clearly zwar Skopus über das Modalverb nehmen, aber es existiert keine Lesart, bei der clearly Skopus hat über das vor dem Modalverb positionierte Adverb. Diese Daten erfahren eine einfache Erklärung, wenn man Bewegung des Modalverbs annimmt. (58b) hat demnach die folgende Struktur:

(58) b'. [IP Gary 2 can $_{1}$ [apparently [VP $t_{1}\left[\operatorname{PredP}_{2} \mathrm{t}_{2}\right.$ lift 100 pounds]]]]

Das Adverb k-kommandiert die Grundposition des Modalverbs. Skopus setzt K-Kommando voraus. Es gibt daher eine Lesart mit Skopus des Adverbs über das Modalverb. Entsprechendes gilt für (58c): clearly kkommandiert die Spur von can. Aber es gibt natürlich keine Spur von already im K-Kommando-Bereich von clearly. Daher existiert keine Lesart mit Skopus von clearly über already.

Nimmt man also an, daß Modalverben und finite Auxiliare bewegt werden, ist für (56) die folgende Struktur anzusetzen:

(56') $\mathrm{She}_{2}\left[\mathrm{I}^{\prime}\right.$ will $_{1}$ [probably $\left[\mathrm{VP}_{1} \mathrm{t}_{1}\left[\mathrm{VP}\right.\right.$ have $\left[\mathrm{PredP}_{2} \mathrm{t}_{2}\right.$ answered the reporter's questions]]]] Es ist damit klar, daß in (56) alle Bedingungen für Satzadverbiale erfüllt sind. Insbesondere k-kommandiert das Satzadverbial das finite Verb. Ein Satzadverbial kann aber nur nach einem Modaladverbial oder nach einem finiten Auxiliar stehen. Es kann nicht nach einem infiniten Auxiliar stehen:

(59) *He has been unfortunately reading the book

Die Erklärung für die Ungrammatikalität von (59) ist dieselbe wie die Erklärung für das obige deutsche Beispiel (53c), nämlich fehlendes K-Kommando des Adverbials über das finite Verb:

(53) c'. *Glücklicherweise viel gelacht wird in diesem Land

Das Adverbial in (59) k-kommandiert ebenfalls nicht das finite Verb oder ein Element von dessen Bewegungskette. Nur das finite Auxiliar oder ein Modalverb werden im Englischen aus einer tieferen Position in 
eine höhere Position bewegt.

In (55) ist auch angegeben, daß ein Satzadverbial nicht möglich ist am Ende des Satzes. Dies gilt dann, wenn das Satzadverbial in den Satz integriert sein soll. Ein Satzadverbial ist am Ende des englischen Satzes nur möglich, wenn es durch eine 'Komma-Intonation' abgesetzt wird.

Um dies zu verstehen, müssen wir nochmal unsere Bedingung betrachten, daß ein Satzadverbial das finite Verb bzw. ein Element seiner Bewegungskette k-kommandieren muß. Diese Bedingung kann ein wenig genauer formuliert werden. Die strukturelle Repräsentation der Finitheit geschieht im Englischen durch die I-Kategorie. Finite Hilfsverben und Modalverben bewegen sich nach I, finite Hauptverben sind mit der I-Kategorie durch eine abstraktere Abhängigkeitsbeziehung verbunden. In all diesen Fällen wird eine Koindizierungsrelation zwischen der Grundposition des finiten verbalen Elements und der I-Position angenommen. Wenn ein Satzadverbial ein Element der Bewegungskette des finiten Verbs k-kommandiert, k-kommandiert es damit auch ein Element der Abhängigkeitskette zwischen I und dem finiten Verb. Die Bedingung für Satzadverbiale kann daher genauer lauten, daß Satzadverbiale ein Element der Abhängigkeitskette zwischen I und dem finiten Verb k-kommandieren müssen. In dieser Formulierung wird deutlich, daß das Bezugselement für Satzadverbiale die IKategorie ist. Wenn ein Adverbial eine spezielle $\mathrm{X}^{0}$-Kategorie als Bezugselement hat, dann muß es sich in dessen Lizenzierungsdomäne befinden. Lizenzierungsdomänen von $\mathrm{X}^{0}$ haben eine bestimmte Richtung. Wie oben ausgeführt, ist die Lizenzierungsdomäne von $\mathrm{V}^{0}$ nach rechts. Funktionale Kategorien lizenzieren stets und in allen Sprachen nach links. Der Lizenzierungsbereich von $\mathrm{I}^{0}$ ist demnach nach links. Daher können Satzadverbiale nicht nach rechts, sondern nur nach links adjungiert werden. Es ergibt sich aus diesem Grund die Ungrammatikalität des Satzadverbials am Satzende in (55).

Betrachten wir als nächstes die Abfolge verschiedener Typen von Satzadverbialen untereinander. Sie ist im Englischen gleich wie im Deutschen. Abfolgebeschränkungen ergeben sich einfach daraus, daß bestimmte Satzadverbiale andere in ihren Skopus nehmen können, während dies umgekehrt nicht möglich ist. So ergibt es z.B. einen Sinn, eine durch ein epistemisches Adverb wahrheitsbewertete Proposition noch durch ein evaluatives Verb wie leider, glücklicherweise zu bewerten. Es ist jedoch kaum sinnvoll, eine bereits emotional bewertete Situation noch einer epistemischen Bewertung zu unterziehen:

(60) a. daß Petra leider wahrscheinlich verreist ist

b. *daß Petra wahrscheinlich leider verreist ist

Dies korrespondiert mit den für das Englische beobachteten Regularitäten für Satzadverbiale (s. bereits Jackendoff 1972:87-93, Travis 1988:186). Adverbien wie z.B. luckily und probably können zwar jeweils in den gleichen syntaktischen Positionen auftreten, jedoch ist eine strikte relative Abfolge festgelegt, wenn beide auftreten:

(61) a. She (luckily/probably) has (luckily/probably) got a job

b. She luckily has probably got a job

c. *She probably has luckily got a job

Kommen wir nun zu den sog. subjektorientierten Adverbialen wie in folgenden Beispielen: 
(62) a. weil Otto klugerweise geschwiegen hat

b. John cleverly made no reply

Diese sind besonders vom semantischen Blickpunkt interessant, da sie eine gewisse Janusköpfigkeit aufweisen. Zum einen drücken sie eine Bewertung eines Tatbestandes durch den Sprecher aus, zum anderen wird diese Bewertung aber prädiziert über das Subjekt des Satzes: es war klug von Otto, daß er geschwiegen hat.

Obwohl eine ganze Reihe von Autoren hier auch einen Propositionsbezug sehen und die subjektorientierten Adverbiale deswegen zu den Satzadverbialen rechnen, ist dies keineswegs unumstritten und in der Literatur findet sich auch die gegenteilige Auffassung. 13

In Frey/Pittner (i.E.) haben wir die Auffassung vertreten, daß es sich beim subjektorientierten Adverbial um einen Propositionsbezug handelt. Da sich der Propositionsbezug von Satzadverbien syntaktisch darin niederschlägt, daß die Grundposition von Satzadverbien das finite Verb k-kommandieren muß, bestätigen die folgenden Daten diese Zuordnung:

(63) a. *Klugerweise geschwiegen hat Otto. ${ }^{14}$

b. *Wahrscheinlich geschwiegen hat Otto.

Diese Beispiele zeigen, daß sich die subjektorientierten Adverbiale hinsichtlich der Positionierung im komplexen Vorfeld wie Satzadverbiale verhalten. Dagegen unterscheiden sie sich deutlich von den sogenannten Adverbialen der Subjekthaltung, die ebenfalls einen Bezug auf das Subjekt aufweisen (in Ausnahmefällen auch auf ein anderes ranghöchstes Argument, das den Träger der Haltung bezeichnet):

(64) a. Gerne gearbeitet hat Otto schon immer.

b. Freiwillig kommen wird er wohl kaum.

c. *Arroganterweise kandidieren will er wohl schon

Da wir einen engen Zusammenhang zwischen möglichen syntaktischen Positionen und semantischen Bezugsbereich annehmen, erwarten wir, daß die Stellungsdaten im Englischen weiteren Aufschluß über die Frage des Propositionsbezugs der subjektorientierten Adverbiale liefern können. Wir überprüfen somit ihre Positionierung relativ zum finiten Verb:

(65) ??He has been cleverly answering their questions.

So bemerken Thomason/Stalnaker (1973), daß carefully sowohl in der A\&W-Lesart als auch in der subjektorientierten Lesart kein Satzmodifikator sei. Allerdings sind eine Reihe von Umformungen möglich, die als Evidenz für einen Propositionsbezug der subjektorientierten Adverbiale gedeutet werden, so etwa, daß die entsprechenden Adjektive sententiale Komplemente zulassen (cf. Peterson 1997:18, der dies für Prädikate mit Ereignisbezug ausschließt):

(i) Daß Hans nicht da war, war sehr nachlässig.

(ii) *Daß Hans sang, war sehr laut.

Es existiert jedoch tatsächlich eine Umformung, die für die übrigen Satzadverbien, nicht jedoch für die subjektorientierten Adverbiale möglich ist, nämlich die Einsetzung in einen Matrixsatz der Form es ist ADV wahr, daß...

(iii) es ist leider/wahrscheinlich/glücklicherweise/*arroganterweise wahr, daß...

Dies spricht dafür, daß die subjektorientierten Adverbiale sich nicht auf unanalysierte Propositionen beziehen, sondern dafür sensitiv sind, wie die Proposition intern beschaffen ist. Subjektorientierte Adverbiale stellen in dieser Hinsicht bestimmte Anforderungen, wie z.B. das Vorhandensein eines Agens. 
Frey/Pittner - 21 -

(65) kann als Beleg dafür gesehen werden, daß auch subjektorientierte Adverbiale im Englischen die Grundposition des finiten Verbs k-kommandieren müssen. Aufgrund des engen Zusammenhangs zwischen Position und semantischem Bezugsbereich kann dies als Evidenz für den Propositionsbezug dieses Adverbialtyps gewertet werden.

Wie schon ausgeführt, unterscheiden sich die subjektorientierten Adverbiale von anderen Satzadverbialen durch ihre besondere Relation zum Subjekt, da sie eine Prädikation über das Subjekt beinhalten. In Frey/Pittner (i.E.) haben wir argumentiert, daß sich im Deutschen die semantische Abhängigkeit eines subjektorientierten Satzadverbials vom Subjekt darin niederschlägt, daß dieses Adverbial (bzw. ein Element seiner Bewegungskette) auf der Oberfläche vom Subjekt k-kommandiert wird. Entsprechendes gilt für das Englische, auch hier spiegelt sich die eingangs schon erwähnte semantische Janusköpfigkeit in der syntaktischen Positionierung wider. Das syntaktische Korrelat des Subjektbezugs dieser Adverbiale liegt in ihrer Grundposition unterhalb der Oberflächenposition des Subjekts in Spec von IP. Dies kann durch 'that-trace'-Effekte gezeigt werden.

Subjektorientierte Adverbiale können wie die anderen Satzadverbiale am Anfang des englischen Satzes stehen:

(66) a. Unfortunately John made no reply

b. Cleverly John made no reply

Das Satzadverbial in (66a) kann nach (2(ii)) in seiner Oberflächenposition basisgeneriert werden. Dies kann entsprechend zu den Überlegung in Abschnitt 5 bestätigt werden durch den Nachweis, daß es zur Aufhebung des 'that-trace'-Effekts bei Bewegung des eingebetteten Subjekts führt:

(67) Who ${ }_{1}$ do you think that unfortunately $t_{1}$ made no reply

Entscheidend ist nun, daß Entsprechendes nicht möglich ist bei einem subjektorientierten Satzadverbial:

(68) * Who ${ }_{1}$ do you think that cleverly $\mathrm{t}_{1}$ made no reply

Nach den Ausführungen in Abschnitt 5 ist cleverly somit in (68) in seiner Oberflächenposition nicht basisgeneriert. Es hat diese Position durch Bewegung erreicht und kann deshalb den 'that-trace'-Effekt nicht aufheben. Wir haben für einen Satz wie (66b) somit eine Repräsentation wie die folgende anzusetzen:

(66)' b. Cleverly John $_{2} \mathrm{t}_{1} \mathrm{t}_{2}$ made no reply

Damit ist gezeigt, daß für ein subjektorientiertes Adverbial des Englischen die gleichen Bedingungen gelten wie für eines des Deutschen: Das subjektorientierte Adverbial (d.h. ein Element seiner Bewegungskette) wird vom Subjekt (d.h. einem Element von dessen Bewegungskette) auf der Oberfläche k-kommandiert. Das Adverbial kkommandiert als Satzadverbial aber in seiner Grundposition die Grundposition des Subjekts und die Grundposition des finiten Verbs. ${ }^{15}$

15 Die Oberflächenorientiertheit teilt das subjektorientierte Satzadverbial mit den Adverbialen der Subjekthaltung. Man findet im Englischen daher bei subjektorientierten Satzadverbialen die gleiche Passivsensitivität wie bei Adverbialen der Subjekthaltung (s. oben Abschnitt 4). Ein subjektorientiertes Satzadverbial bezieht sich somit im englischen Passivsatz auf das Passivsubjekt. Wiederum finden wir den Un- 


\section{Zur Frage der Rechtsadjunktion}

Das Auftreten von Adverbialen am rechten Satzrand des englischen Satzes, das wir in unseren bisherigen Überlegungen weitgehend ausgeklammert haben, wirft einige äußerst schwierige Fragen auf.

Es gibt Eigenschaften von Adverbialen in satzfinaler Position, die elegant durch Rechtsadjunktion erfaßt werden. Zunächst läßt sich beobachten, daß Adverbiale in den finalen Positionen des englischen Satzes umgekehrt zu der Reihenfolge auftreten, wie wir sie für das deutsche Mittelfeld gezeigt haben. So nennen Quirk/Greenbaum (1973:241) z.B. die Abfolge A\&W-Adverbial > Lokaladverbial > Temporaladverbial und geben dazu folgendes Beispiel:

(69) He was working with his shears in the garden the whole morning.

Bei anderen Abfolgen ergibt sich zwar keine Ungrammatikalität, aber diese Abfolgen werden als weniger normal empfunden. Umstellungen können durch die Länge der Konstituenten bedingt sein oder vorgenommen werden, um Endfokus zu erreichen. Abstrahiert man von diesen Faktoren, zeigen sich durchweg die umgekehrten Abfolgen wie im deutschen Mittelfeld. So findet man z.B. in der Literatur auch den Hinweis, daß am Satzende Adverbiale des Grundes oder des Zweckes ebenfalls nach den Lokaladverbialen auftreten. Dies widerspricht der Position von Cinque (1997), der annimmt, daß im Englischen Adverbiale wie in (69) und Adverbiale der Art und Weise, des Grundes oder des Zweckes nach den Objekten ungeordnet seien.

Die spiegelbildliche Abfolge der Adverbiale am Satzende im Vergleich zur Abfolge im präverbalen Bereich ist in einer Analyse mit Rechtsadjunktion erwartet. Insbesondere können die unmarkierten Abfolgen in einer Analyse mit Rechtsadjunktion mit unseren Bedingungen in (2) unmittelbar erklärt werden.

Auch die Skopusverhältnisse lassen sich leicht mit Rechtsadjunktion erfassen. Es läßt sich beobachten, daß in satzfinaler Position Adverbiale Skopus über ein anderes vorangehendes Adverbial in satzfinaler Position haben:

(70) a. I hit him twice on two occasions (eindeutig: zweimal bei jeder der beiden Möglichkeiten)

b. She has made extra trips frequently willingly (eindeutig: willingly hat Skopus über frequently)

c. She has made extra trips willingly frequently (eindeutig: frequently hat Skopus über willingly)

d. They've done it twice on purpose for years (eindeutig: for years hat Skopus über on purpose, dieses hat Skopus über twice)

e. He attended classes at at least one university nearly every day of the week (eindeutig: nearly every day ... hat Skopus über at least one university)

Diese Daten ergeben sich unmittelbar unter Rechtsadjunktion, da bei dieser Analyse das weiter rechts stehende Adverbial das direkt vorangehende Adverbial asymmetrisch k-kommandiert.

terschied zum Deutschen. Im Deutschen bezieht sich das subjektorientierte Satzadverbial im Passivsatz auf das 'Tiefensubjekt', d.h. auf das implizite Argument. Die Erklärung für diesen Unterschied ist die gleiche, wie wir sie für den entsprechenden Unterschied bei den Adverbialen der Subjekthaltung in Abschnitt 4 gegeben haben. 
Allerdings gibt es empirische und konzeptuelle Einwände gegen Rechtsadjunktion. Bereits Larson (1988) ging davon aus, daß alle Adverbiale, wenn sie nach den Objekten auftreten, von den Objekten k-kommandiert werden. Desweiteren gibt es einflußreiche Arbeiten (Kayne 1994, Haider 1993), die dafür argumentieren, daß syntaktische Strukturen prinzipiell nur binär rechtsverzweigend sein können. Rechtsadjunktionen können danach in der syntaktischen Struktur nicht existieren.

Für Larson waren es hauptsächlich Bindungsdaten und andere Indikatoren für K-Kommando-Beziehungen, die zu der Annahme führten, daß die Adverbiale am Satzende nicht rechtsadjungiert sind:

(71) a. She visited every colleague ${ }_{1}$ on his ${ }_{1}$ birthday

b. I shot the men willingly near each other's home

c. She took none of them on purpose for any reason I am aware of

Die Daten zeigen, daß sich die Adverbiale am Satzende in der K-Kommando-Domäne des Objekts befinden. Larson positioniert die Adverbiale am Satzende ebenso wie die Argumente in einer vom Hauptverb aufgespannten VP-Schalenstruktur. Vorangehende Phrasen k-kommandieren dann weiterhin asymmetrisch die nachfolgenden Phrasen. Auch Adverbiale am Satzende sind somit in einer binär rechtsverzweigenden Struktur angeordnet.

Unter Larsons Annahmen lassen sich Skopusdaten wie in (70) aber nicht erfassen. Da das vorangehende Adverbial das nachfolgende k-kommandiert, würde man unter dieser Analyse genau die umgekehrten Skopusverhältnisse erwarten. Auch eine LF-Bewegungsanalyse scheint unter Larsons Annahmen nicht möglich. Um die Eindeutigkeit der Beispiele in (70) zu gewährleisten, wären eine Reihe sehr willkürlicher Annahmen notwendig.

Cinque (1997) schlägt für Sätze wie in (70) eine Basisstruktur vor, deren K-Kommando-Beziehungen den Skopusfakten entspricht. Die Adverbiale werden mit der richtigen K-Komando-Beziehung vor dem Verb basisgeneriert. Die Oberfläche wird dann durch eine neuartige Bewegung abgeleitet ('Intraposition'). Dem Satz (70b) z.B. liegt die folgende Abfolge zugrunde:

(72) She has [willingly [frequently [made extra trips]]]

Daraus wird zunächst abgeleitet:

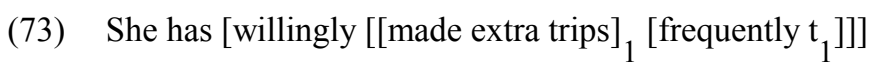

Die Oberfläche entsteht durch eine weitere Intraposition:

(74) She has $\left[\left[[\text { made extra trips }]_{1}\left[\text { frequently } t_{1}\right]\right]_{2}\left[\right.\right.$ willingly $\left.\left.t_{2}\right]\right]$

Ein Problem für diesen Vorschlag ist sicherlich, daß Cinque für diese Bewegungen und für ihre Zielpositionen keine Theorie bereitstellt. Das Hauptproblem aber ist, daß Bindungsdaten wie in (71) mit diesem Ansatz nicht erfaßt werden können. Da das Objekt innerhalb einer komplexeren Konstituente vor das Adverbial bewegt wird, kann das Objekt ein nachfolgendes Adverbial nicht k-kommandieren.

Somit liegt eine widersprüchliche Datenlage vor. Die Reihenfolgebedingungen und die Skopusdaten sprechen für eine Rechtsadjunktion der Adverbiale am Satzende. Unter Rechtsadjunktion könnten die Daten 
desweiteren unmittelbar mit den Bedingungen in (2) erfaßt werden. Die Bindungsdaten hingegen sprechen dafür, alle satzfinalen Adverbiale strukturell zumindest tiefer als das Objekt zu positionieren. Konzeptuelle Überlegungen sprechen dafür, generell nur rechtsverzweigende (Basis)-Strukturen zuzulassen.

Wenn man die konzeptuellen Überlegungen nicht übernehmen möchte, wäre eine Möglichkeit, dieses Dilemma zu überwinden, K-Kommando als Voraussetzung für Bindungsbeziehungen aufzugeben. Stattdessen müßten die Bedingungen für Bindung, die Bedingung für die Lizenzierung negativer Polaritätselemente usw. über Präzedenz formuliert werden (so z.B. Jackendoff 1990a). Man könnte dann an einer Analyse der satzfinalen Adverbiale mit Rechtsadjunktion festhalten. Dies würde aber tiefgreifende und weitreichende Veränderungen der Theorie beinhalten.

Wir möchten diesen weitreichenden Schritt nicht machen und werden im folgenden einen anderen Weg skizzieren. Chomsky (1995: 333) bemerkt bezüglich der Phrasen nach den Objekten: "if a shell structure is relevant at all, the additional phrases might be supported by empty heads below the main verb...". Diese leeren Köpfe wären keine Spuren des Verbs. Die Grundposition des Verbs wäre vielmehr beim tiefsten Objekt. Die leeren Köpfe würden eine rechtsverzweigende Struktur für die satzfinalen Adverbiale im K-Kommando-Bereich der Objekte aufspannen. Man könnte nun annehmen, daß diese leeren Köpfe zwar eine syntaktische Positionierung der Adverbiale ermöglichen, daß die Adverbiale aber in dieser Struktur nicht interpretiert werden können, da die leeren Köpfe selbst semantisch nicht interpretiert werden. Damit die satzfinalen Adverbiale interpretiert werden können, müssen sie verbunden werden mit abstrakten Modifikationsmarkern, die im interpretierten Satzrahmen positioniert werden müssen, d.h. in dem Bereich, der vom Verb und den darüber liegenden Köpfen aufgespannt wird. Das Konzept der Modifikationsmarker erinnert an das Konzept der Skopusmarker von Williams (1986). Die Modifikationsmarker kennzeichnen für ein gegebenes Adverbial den Knoten in der syntaktischen Struktur, der von dem Adverbial modifiziert wird. Aufgrund der inhärenten Semantik eines Adverbials ist eindeutig, welcher der in (2) genannten Klassen es zuzuordnen ist. Ein Modifikationsmarker wird gemäß der Bedingungen in (2) gesetzt.

Entscheidend wäre dann die folgende Bedingung gegen überkreuzende Pfade: Die Zuordnungen der Adverbiale an ihre Marker dürfen in der Basisstruktur nicht überkreuzen. Sind nun für die Positionierungen der Marker im interpretierten Satzrahmen die Konditionen (2) einschlägig, ergibt sich mit der Pfadbedingung unmittelbar die unmarkierte Abfolge der satzfinalen Adverbiale. Wenn Skopus über die Marker berechnet wird, ergeben sich auch die Skopusdaten. Die Bindungsdaten ergeben sich, da die satzfinalen Adverbiale in einer von den Argumenten k-kommandierten rechtsverzweigenden Schalenstruktur basisgeneriert werden.

Man beachte, daß bis auf die Bemerkung zu satzfinalen Satzadverbialen die Überlegungen der vorigen Abschnitte, die von Rechtsadjunktion an höhere Projektionen in der Basistruktur Gebrauch machen, sich direkt in diesem Rahmen umformulieren lassen. Es versteht sich allerdings, daß das Konzept der Modifikationsmarker zum jetzigen Zeitpunkt noch sehr spekulativ ist.

\section{Zusammenfassung}


Unser Ziel war, erste Überlegungen dazu anzustellen, ob sich die in Frey/Pittner (i.E.) beobachteten Regularitäten für die Abfolge von Adverbialen im deutschen Mittelfeld auch im Englischen wiederfinden. Unser Grundgedanke dabei ist, daß diese, da es sich um Widerspiegelungen der semantischen Verhältnisse handelt, auch im Englischen gelten müssen und daß sich Unterschiede in der Adverbialstellung auf unabhängig davon existierende Unterschiede in der Satzstruktur zurückführen lassen. Wir haben versucht zu zeigen, daß die in (2) formulierten Bedingungen, die ursprünglich anhand des Deutschen entwickelt wurden, auch im Englischen wirksam sind. Dies wurde zunächst überprüft für Adverbiale der Art und Weise. Deren scheinbare große Stellungsfreiheit im Englischen ist durch eine Adjunktion an $\mathrm{V}^{0}$ und Larsonische Verbbewegungen bedingt. Auch die Adverbiale der Subjekthaltung, die die mentale Einstellung eines Referenten zum Vorgang beinhalten, unterliegen in beiden Sprachen denselben Bedingungen. Orientierungsunterschiede bei diesen Adverbialen im Deutschen und Englischen ließen sich mit unterschiedlichen strukturellen Positionen des Subjekts im deutschen und englischen Satz in Verbindung bringen. 'That-trace'-Effekte stützen die Annahme, daß z.B. Temporaladverbiale im Englischen ihre Grundposition oberhalb des Subjekts, Lokaladverbiale z.B. jedoch ihre Grundposition unterhalb des Subjekts einnehmen, wobei dies jeweils den Verhältnissen im Deutschen entspricht. Satzadverbiale haben Propositionsbezug. Für sie gilt im Englischen wie im Deutschen, daß sie die Grundposition des finiten Verbs k-kommandieren müssen. Dies gilt auch für die subjektorientierten Satzadverbiale, die sich jedoch von anderen Satzadverbialen durch ihre besondere Relation zum Subjekt unterscheiden. Diese findet im Englischen wie auch im Deutschen ihren syntaktischen Niederschlag darin, daß diese Adverbiale ihre Grundposition unterhalb der Oberflächenposition des Subjekts einnehmen, was für das Englische wiederum anhand der 'that-trace'-Daten gezeigt werden konnte.

\section{Literatur}

Abraham, Werner (1986): Word order in the middle field of the German sentence. In: W. Abraham/S. de Meij (eds.), Topic, focus and configurationality. Amsterdam: Benjamins, 15-38.

Alexiadou, Artemis (1997): Adverb placement. A case study in antisymmetric syntax. Amsterdam: Benjamins.

Bartsch, Renate (1972): Adverbialsemantik. Die Konstitution logisch-semantischer Repräsentationen von Adverbialkonstruktionen. Frankfurt: Athenäum.

Bierwisch, Manfred (1988): On the Grammar of Local Prepositions: In: Bierwisch, M., Motsch, W. \& Zimmermann, I. (eds.): Syntax, Semantik und Lexikon. Studia Grammatica XXIX. Berlin: Akademie-Verlag, $1-65$.

Browning, M.A. (1996): CP recursion and 'that-t' effects. Linguistic Inquiry 27, 237-255.

Bowers, John (1993): The Syntax of Predication. Linguistic Inquiry 24, 591-656

Cinque, Gulielmo (1997): Adverbs and functional heads. A cross-linguistic perspective. Erscheint bei: Oxford University Press.

Chomsky, Noam (1981): Lectures on Government and Binding. Dordrecht: Foris

Chomsky, Noam (1995): The Minimalist Program. Cambridge (Mass.): MIT Press

Costa, Jo J o (1996): Adverb Positioning and V-movement in English. Studia Linguistica 50, 22-34.

Ernst, Thomas (1991): On the Scope Principle. Linguistic Inquiry 22, 750-756.

Ernst, Thomas (1998a): On the Scopal Basis of Adjunct Licensing. Erscheint in: Proceedings of NELS 1998.

Ernst, Thomas (1998b): Why I believe in right adjunction. Manuskript Rutgers University.

Frey, Werner (1993): Syntaktische Bedingungen für die semantische Interpretation. Über Bindung, implizite 
Argumente und Skopus. Berlin: Akademie-Verlag. (studia grammatica XXXV)

Frey, Werner/Karin Pittner (i.E.): Zur Positionierung der Adverbiale im deutschen Mittelfeld. Erscheint in: Linguistische Berichte.

Grundzüge einer deutschen Grammatik (1981): Von einem Autorenkollektiv unter der Leitung von K.-E. Heidolph, W. Flämig und W. Motsch. Berlin: Akademie-Verlag.

Haider, Hubert (1993): Deutsche Syntax - Generativ. Tübingen: Narr.

Jackendoff, Ray S. (1972): Semantic interpretation in generative grammar. Cambridge/Mass.: MIT Press.

Jackendoff, Ray S. (1990a): On Larson's Analysis of the Double Object Construction. Linguistic Inquiry 21, 427-456.

Jackendoff, Ray S. (1990b): Semantic Structures. Cambridge/Mass.: MIT Press.

Kayne, Richard (1994): The Antisymmetry of Syntax. Cambridge (Mass.): MIT Press.

Kiss, Katalin É. (1996): Two Subject Positions in English. The Linguistic Review 13, 119-142

Larson, Richard (1988): On the double object construction. Linguistic Inquiry 19, 335-391

Lenerz, Jürgen (1977): Zur Abfolge nominaler Satzglieder im Deutschen. Tübingen: Narr. (Studien zur deutschen Grammatik 5)

Maienborn, Claudia (1996): Situation und Lokation. Die Bedeutung lokaler Adjunkte von Verbalprojektionen. Tübingen: Stauffenburg.

McCawley, James D. (1983): The syntax of some English adverbs. Proceedings of the 19th Regional Meeting of the Chicago Linguistic Society. Chicago: University Press, 263-282.

Peterson, Philip L. (1997): Fact proposition event. Dordrecht: Kluwer.

Pittner, Karin (1996): Adverbiale im Deutschen. Untersuchungen zu ihrer Stellung und Interpretation. Habilitationsschrift Universität Stuttgart. (erscheint beim Stauffenburg Verlag, Tübingen)

Quirk, Randolph/Sidney Greenbaum (1973): A university grammar of English. London: Longman.

Rizzi, Luigi (1995): The Fine Structure of the Left Periphery. Ms.

Roberts, Ian (1987): The Representation of Implicit and Dethematized Subjects. Dordrecht: Foris.

Speas, Margaret (1990): Phrase Structure in Natural Language. Dordrecht: Kluwer.

Thomason, Richard/Robert Stalnaker (1973): A semantic theory of adverbs. Linguistic Inquiry 4, 195-220.

Travis, Lisa (1988): The syntax of adverbs. McGill Working Papers in Linguistics. Special Issue on Comparative Germanic Syntax. McGill University, 280-310.

Williams, Edwin (1986): A Reassignment of the Functions of LF. Linguistic Inquiry 19, 135-146.

Werner Frey und Karin Pittner

(pittner@ims.uni-stuttgart.de)

August 1998 